\title{
Tractography and the connectome in neurosurgical treatment of gliomas: the premise, the progress, and the potential
}

\author{
Fraser Henderson Jr., MD, ${ }^{1,3}$ Kalil G. Abdullah, MD, ${ }^{4}$ Ragini Verma, PhD, ${ }^{1,2}$ and Steven Brem, MD ${ }^{1}$ \\ 1Department of Neurosurgery, Perelman School of Medicine, University of Pennsylvania; ${ }^{2 D i C I P H R ~(D i f f u s i o n ~ a n d ~ C o n n e c t o m i c s ~}$ \\ in Precision Healthcare Research) Lab, Department of Radiology, Perelman School of Medicine, University of Pennsylvania, \\ Philadelphia, Pennsylvania; '3epartment of Neurosurgery, The Medical University of South Carolina, Charleston, South Carolina; \\ and ${ }^{4}$ Department of Neurosurgery, University of Texas Southwestern Medical Center, Dallas, Texas
}

The ability of diffusion tensor MRI to detect the preferential diffusion of water in cerebral white matter tracts enables neurosurgeons to noninvasively visualize the relationship of lesions to functional neural pathways. Although viewed as a research tool in its infancy, diffusion tractography has evolved into a neurosurgical tool with applications in glioma surgery that are enhanced by evolutions in crossing fiber visualization, edema correction, and automated tract identification. In this paper the current literature supporting the use of tractography in brain tumor surgery is summarized, highlighting important clinical studies on the application of diffusion tensor imaging (DTI) for preoperative planning of glioma resection, and risk assessment to analyze postoperative outcomes. The key methods of tractography in current practice and crucial white matter fiber bundles are summarized. After a review of the physical basis of DTI and post-DTI tractography, the authors discuss the methodologies with which to adapt DT image processing for surgical planning, as well as the potential of connectomic imaging to facilitate a network approach to oncofunctional optimization in glioma surgery. https://thejns.org/doi/abs/10.3171/2019.11.FOCUS19785

KEYWORDS connectomics; DTI; diffusion tensor imaging; fiber tractography; glioma surgery

$\mathrm{C}$ LINICAL tractography emerged at the beginning of this century with the discovery that noninvasive MR diffusion imaging can approximate human neuronal pathways in vivo. Yet neurosurgeons today lack a standardized method for acquiring and using reconstructed estimations of white matter tracts in glioma surgery for several reasons: the heterogeneity of user-dependent characteristics; the difficulty in validating the correlation between imaging and function; and the confusing variety of diffusion tensor imaging (DTI) and post-DTI algorithms available at a few academic centers. In preparation for surgery, surgical teams specify regions of interest (ROIs) with resultant user variability ${ }^{61}$ Other problems that affect the interpretation and accuracy of DTI include false continuities, crossing fibers, fiber truncation, and edema "noise." ${ }^{34}$
These limitations have inspired solutions like anatomically constrained tracking as well as a proliferation of "multitensor" and "non-tensor" alternatives to combat the voxel limitations of DTI. ${ }^{42,61}$

The Human Connectome Project, a publicly funded, multiinstitutional advanced diffusion imaging effort, reflected early enthusiasm for the potential of diffusion tractography to inform neuroscience through enhanced awareness of white matter connections, and one fruit of that project has been a landmark atlas of human brain connectivity. ${ }^{9}$ Yet whereas the advanced imaging acquired for that study remains beyond the scope of everyday clinical feasibility, the clinically feasible method of DTI has become a prevalent paradigm in glioma surgery. We therefore review current methods of DTI validation,

ABBREVIATIONS AF = arcuate fasciculus; $C S D=$ constrained spherical deconvolution; $C S T=$ corticospinal tract; $\mathrm{DSI}=$ diffusion spectrum imaging; $\mathrm{DTI}=$ diffusion tensor imaging; FA = fractional anisotropy; FACT = fiber assignment by continuous tracking; fMRI = functional MRI; GQI = generalized Q-sampling imaging; GTR = gross-total resection; HARDI = high angular resolution diffusion imaging; HDFT = high-definition fiber tractography; HGG = high-grade glioma; IFOF = inferior fronto-occipital fasciculus; $\mathrm{KPS}=$ Karnofsky Performance Scale; $\mathrm{LGG}=$ low-grade glioma; ODF = orientation distribution function; $\mathrm{QA}=$ quantitative anisotropy; $\mathrm{QBI}=\mathrm{Q}$-ball imaging; ROI = region of interest; SDF = spin distribution function; SLF = superior longitudinal fasciculus; UKF = unscented Kalman filter.

SUBMITTED October 1, 2019. ACCEPTED November 13,2019.

INCLUDE WHEN CITING DOI: 10.3171/2019.11.FOCUS19785. 
the underlying fascicular anatomy, and the source of the reference images. We then present a summary of current studies with a view to next-generation tractography for oncofunctional optimization. ${ }^{32}$ In particular, we develop the hypothesis that the use of connectomics, a picture of the networks in the brain, will permit the neurosurgeon to tailor approaches for the individual patient that are least disruptive to vital networks. ${ }^{9,12}$

\section{Discussion}

\section{Projection Fibers, Commissural Fibers, and Association Fibers}

White matter tracts can be categorized as association, projection, or commissural..$^{14}$ Projection fibers interconnect cortical regions with deeper regions (Fig. 1). The corticospinal tracts (CSTs) emanate from the precentral gyrus of the posterior frontal lobe. Fibers enter the posterior limb of the internal capsule between the globus pallidus and thalamus to form the pyramidal tracts.

Commissural tracts interconnect similar cortical regions between opposite hemispheres. They traverse from left to right. The corpus callosum, a large commissural bundle spanning the floor of the interhemispheric fissure beneath the cingulum, is anatomically segmented into the rostrum, genu, body, and splenium. Forceps minor is the portion spanning anteriorly from the genu to the frontal lobes, whereas forceps major spans posteriorly from the splenium to the occipital lobes. The tapetum and anterior commissure connect left and right temporal lobes.

Association tracts interconnect ipsilateral cortical regions. The superior longitudinal fasciculus/arcuate fasciculus white matter complex (SLF/AF) involves transmission of speech and language, with other important associations in arenas of attention, memory, emotion, and visuospatial function. The SLF has 4 portions: SLF I connects the superior parietal lobule and supplemental motor areas of the superior frontal gyrus; SLF II connects the inferior parietal lobule and the dorsolateral prefrontal cortex; SLF III connects the inferior parietal lobule through parietal and frontal opercula to the inferior frontal gyrus; and AF connects the frontal operculum to the temporal operculum. ${ }^{21}$

In broad terms the inferior longitudinal fasciculus (ILF) connects the occipital lobe to the temporal lobe. Damage to it is associated with deficits in object recognition, including prosopagnosia. ${ }^{64}$ The cingulum interconnects medial parts of the frontal, parietal, and temporal lobes. In addition to its limbic role in emotional processing, it connects the anterior cingulate and posterior cingulate cortices, a vital function for the default mode network. ${ }^{11}$ The inferior fronto-occipital fasciculus (IFOF) participates in semantic language processing. Forming the "ventral pathway" within the dual-stream model of language processing, it complements the "dorsal pathway" of the SLF/AF. It probably facilitates goal-oriented behavior because it is involved in the salience and executive networks. ${ }^{22}$ The uncinate fasciculus (UF) connects the temporal pole, uncus, parahippocampal gyrus, and amygdala with the lateral orbitofrontal cortex, cingulum, and frontal pole. It has general associations in semantic processing,
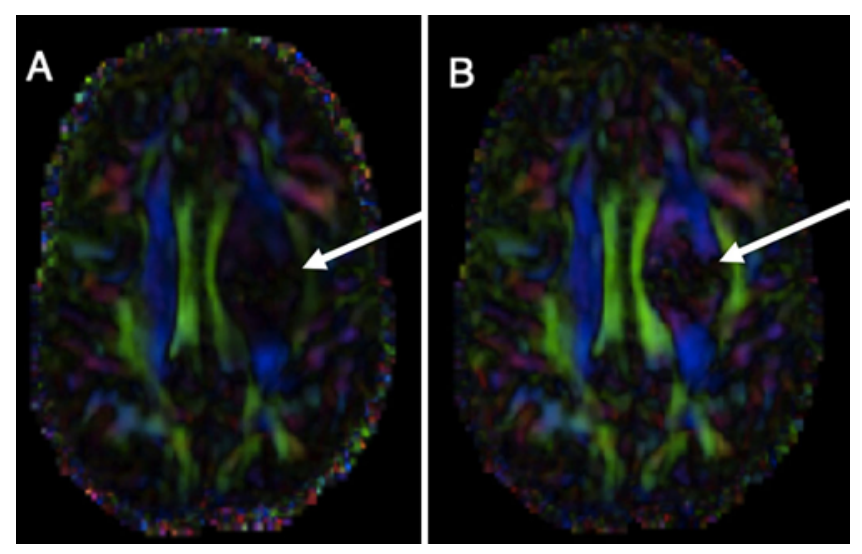

FIG. 1. A: FA color map overlaid on a T1-weighted postcontrast MRI study of a patient with a left-sided brain tumor. Note generally that projection fibers are blue, association fibers are green, and commissural fibers are red. B: An edema correction algorithm has been applied to the same study, further unveiling peritumoral white matter fibers (arrows). Edema invariance processing overcomes a major limitation of peritumoral diffusion-based imaging.

episodic memory, and emotional processing. The frontal aslant tract (FAT), recognized only a decade ago, is a leftlateralized fiber bundle connecting ipsilateral superior and inferior frontal lobe gyri. It is highly integrated with the pars opercularis, and damage to it impairs initiation of speech and verbal fluency. ${ }^{10}$

\section{The Basis of Diffusion MR Images}

Diffusion-based tractography is predicated on the observation that white matter fiber bundles coincide with the direction of maximum water diffusivity. ${ }^{42}$ In anisotropic diffusion, as occurs when water is relatively confined within human axonal architecture, molecules do not diffuse equally in all directions. Axonal membrane and myelin sheathing regiment the Brownian motion of water into a flow parallel with the axons. ${ }^{42}$ Diffusion-based imaging thus exploits anisotropy.

For each voxel in diffusion MR, a diffusion probability density function (this term is used interchangeably with molecular displacement distribution) is derived from signals. This function then provides a synopsis of the total diffusion of the population of water molecules contained in that imaging voxel. ${ }^{37}$ The diffusion behavior within an entire voxel has been approximated by an entity called the diffusion tensor. A tensor is a single, computed result assembled assuming gaussian distributions from the sum contribution of multiple gradient samplings performed by the MRI machine. Six unique variables define a diffusion tensor, explaining the DTI requirement for at least 6 diffusion-encoding directions. Three eigenvalues $(\lambda 1, \lambda 2, \lambda 3)$ represent the apparent diffusivities along the 3 eigenvectors, which describe the major, medium, and minor principal axes of an ellipsoid fitted to the data of a particular voxel. ${ }^{41}$ Thus the diffusion ellipsoid visually represents the diffusion tensor and the ensemble of neural anatomy microstructure within a particular voxel.

The degree of inequality of the diffusion is characterized by the fractional anisotropy (FA) quotient, calculated 


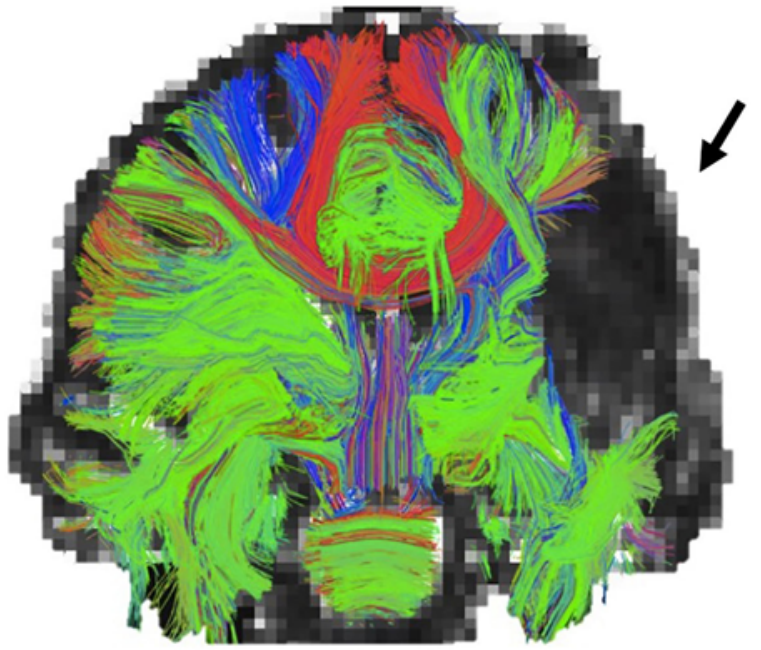

FIG. 2. A coronal view of white matter tracts reveals the problem of peritumoral edema that may foil the ability to determine underlying white matter anatomy by reducing adjacent FA values. In this image the inability to visualize a region of fibers (arrow) occurs because free water conceals the underlying tracts that may contain eloquence. Edema correction/invariance technology seeks to address this limitation of tractography in glioma surgery.

from the standard deviation of the 3 eigenvalues. FA is therefore an index measuring the degree of directionality of intravoxel diffusivity. It ranges from 0 (isotropy, envisioned as a boundless volume of water) to 1 (where diffusion can only occur along a single line). Clinically, white matter tracts are usually identified by following the direction of the principal eigenvector in each voxel with FA values exceeding 0.1-0.3. FA maps take the direction of maximum diffusivity at each voxel and assign the colors red, green, and blue corresponding to the directional plane (Fig. 1). When the primary eigenvalue is much larger than the second and third eigenvalues, FA approaches 1.0, revealing a dominant direction of diffusion. Conversely, low FA values approaching 0.0 are found in white matter with edema that lack a dominant direction of diffusion, or in regions of infiltration that alter the water directionality (Fig. 2).

\section{Tracking}

Tracking (i.e., streamlining) is the process of specifying parameters like FA and angular threshold to MR diffusion information in order to connect voxels into underlying physiological tracts. It is performed in forward and backward directions starting from a seed-point ROI until it reaches a threshold for halting (Fig. 3). Further specification occurs by restricting the tracking to only include fibers also traveling through secondary inclusion ROIs, or to exclude fibers that pass through exclusion ROIs. Angular threshold refers to the maximum bending angle permitted for a tract trajectory, chosen to truncate trajectories before they become anatomically inaccurate. ${ }^{41}$ Thousands of axons may traverse through a single MRI voxel, limiting the resolution with which to estimate potentially complex geometrical configurations of axons, and it can be problematic when fibers cross or branch within an individual voxel ${ }^{42}$ (Fig. 4).
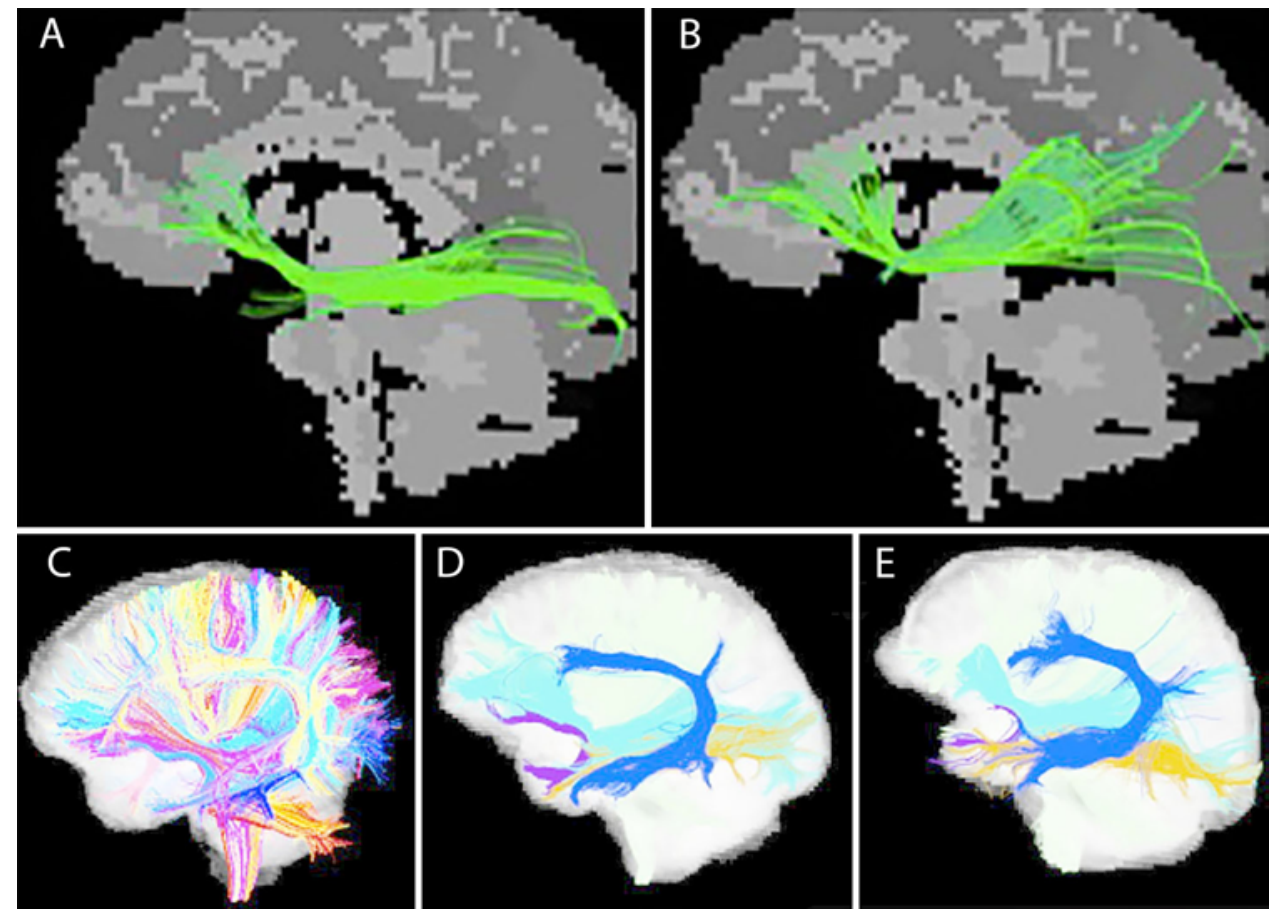

FIG. 3. Tracking or streamlining is the process of extracting meaningful white matter bundles emanating from a seed point or connecting 2 or more ROIs. Different ROI placement by 2 different experts (A vs $\mathbf{B})$ in the same patient may reveal variable depictions of the same tract. Increasingly, automated solutions may reduce variability. Automated tract extraction performed using an annotated fiber clustering atlas (C) has been applied to 2 individual patients ( $D$ and $E$ ), showing preservation of individual variability in the AF (light blue). 

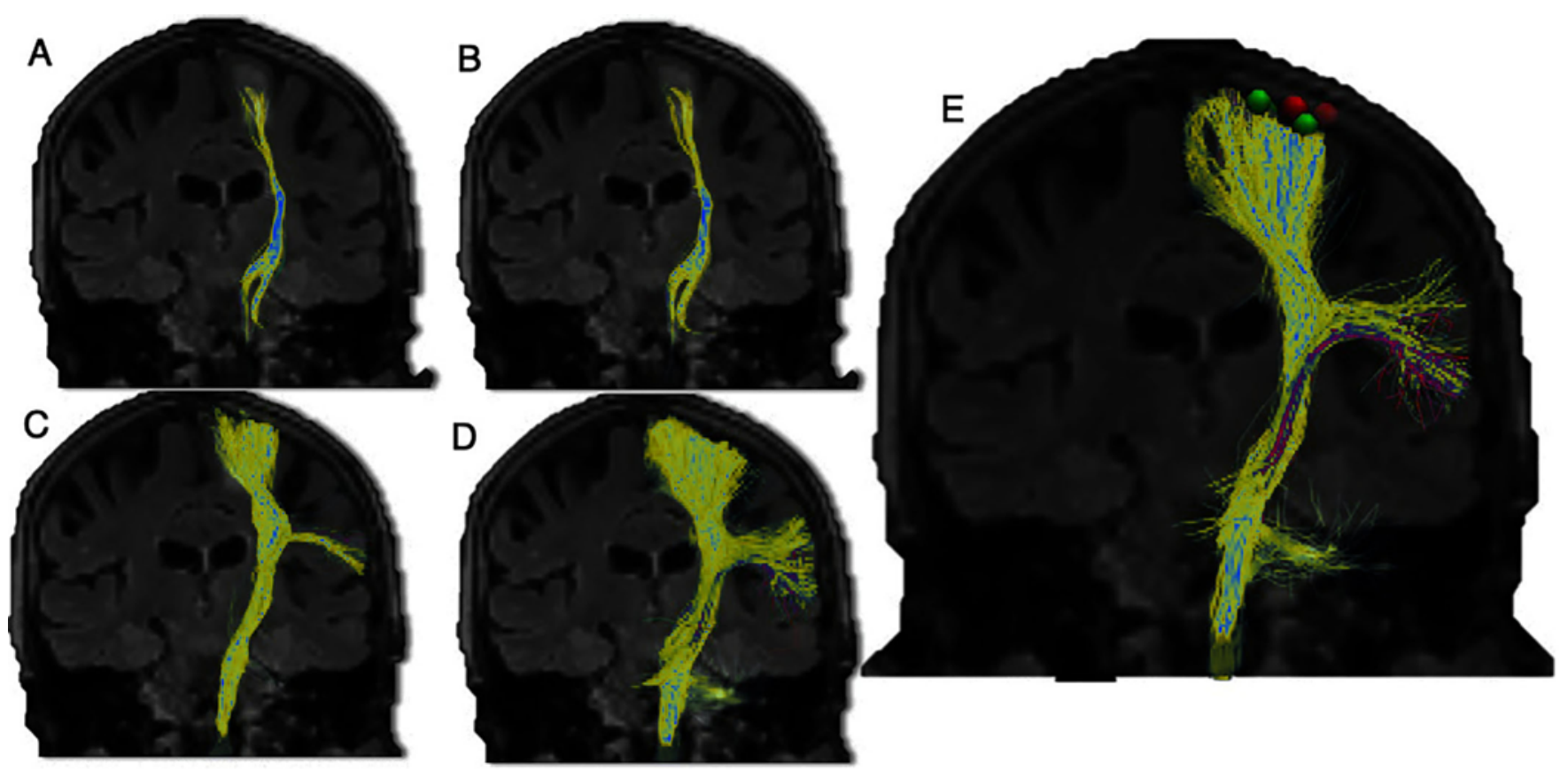

FIG. 4. Tracking of the CST (yellow) reveals greater visualization with successively more advanced tractography methods: with a DTI model (A); with a free-water corrected DTI model, showing a mild increase (B); with a HARDI model and probabilistic tracking (C); and in a multicompartment model with free-water correction and probabilistic tracking (D). The ability to accurately resolve white matter fibers that cross within a voxel has been a limiting factor; the absence of DTI tracts on a postprocessed scan does not preclude the underlying presence of white matter tracts. A variety of solutions like probabilistic tracking and HARDI have been developed to mitigate this limitation. Analysis of stereotactic cortical and subcortical stimulation points (E; e.g., red and green cortical points) in conjunction with tractography represents an important methodology in validation studies.

\section{Deterministic Versus Probabilistic Tracking}

Two approaches to tracking are deterministic and probabilistic. Deterministic methods generate a single fiber connection from a start point: one reconstructed trajectory issues from one seed point. The probabilistic approach detects many possible connections from the start points; it considers that multiple pathways may occur from the seed point and from each point along the path. It does this through calculating algorithms of the probabilities of possible divergences. ${ }^{3,43}$ Probabilistic tracking tends to disperse trajectories more than deterministic methods, thereby delineating a greater portion of the tract, but requiring vast processing power. ${ }^{57}$

The method chosen may depend on the task at hand. For example, DTI has underestimated the anterior extent of Meyer's loop fibers or the face area of the homunculus, and so probabilistic methods are favored.$^{62}$ In general, deterministic methods reduce the portions of invalid connections, whereas probabilistic methods display higher average bundle coverages ${ }^{49}$ (Fig. 4). Despite the prevalence of probabilistic methods in research, much of the initial clinical literature on DTI refers to a deterministic method called "fiber assignment by continuous tracking" (FACT). ${ }^{8}$

\section{Clinical Applications of DTI in Glioma Surgery}

Numerous case series have reported a close correlation between DTI and intraoperative direct stimulation (Table 1). Bello et al. ${ }^{5}$ found strong concordance such that DTI reduced the number of stimulations needed to safely confirm a tract, thereby decreasing patient fatigue and the likelihood of disruptive seizures. Furthermore, tractography in that study proved adept at identifying patients at highest risk of immediate postoperative deficits. In those with postoperative deficits, preoperative DTI had shown the tracts to be qualitatively dislocated or infiltrated. Nevertheless, DTI has not been validated as a substitute for intraoperative stimulation, which remains the gold standard. 4,66

The challenge of validating incremental technological advances in tractography limits their widespread clinical implementation. Whereas animal studies with tracers have been undertaken to validate a few tractography paradigms at a voxel-by-voxel level, validation in patients with human glioma has been performed by multimodal efforts involving motor, language, and visual mapping during awake brain surgery in which direct electrical stimulation (cortical and subcortical) and correlation with functional MRI task activation maps has been used. ${ }^{65}$

An early randomized controlled trial of the use of tractography for glioma showed clear benefits in increasing overall survival, extent of resection, and 6-month Karnofsky Performance Scale (KPS) scores in the DTI-aided high-grade glioma (HGG) group.$^{73}$ The average KPS scores among patients with either low-grade gliomas (LGGs) or HGGs were significantly higher in those who had undergone DTI than in those who had undergone standard neuronavigational guidance of resection: KPS scores overall for patients with gliomas, 86 versus 74, p < 


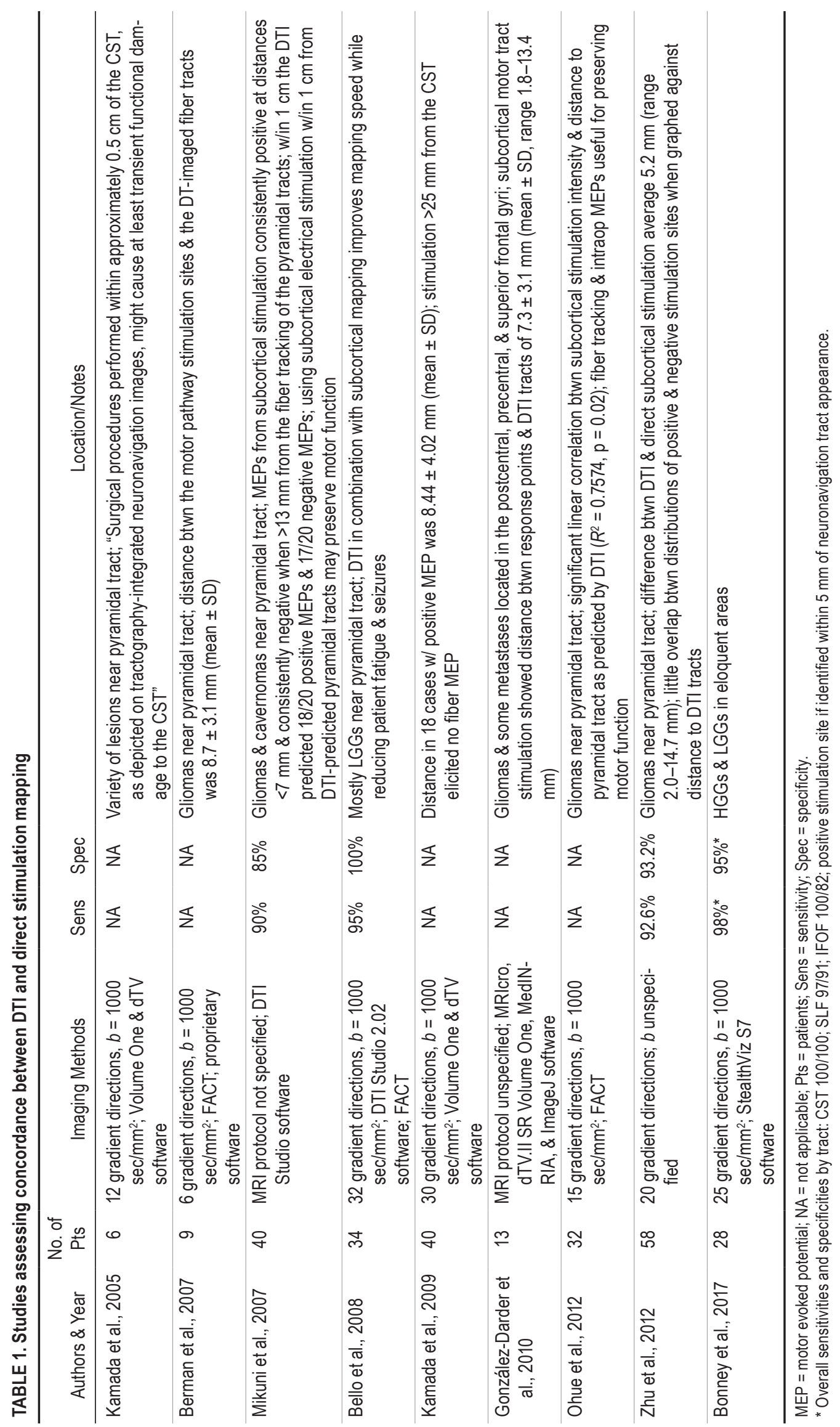


0.001; for those with LGGs, 93 versus 86, $\mathrm{p}=0.013$; and for those with HGGs, 77 versus 53, $\mathrm{p}=0.001$. Overall rates of gross-total resection (GTR) in the DTI group (118 cases, $72 \%$ GTR) were significantly higher than in the control group that underwent standard neuronavigation (120 cases, $51.7 \%$ GTR, p = 0.002). Other benefits can include successful identification of Meyer's loop tracts with decreased incidence of postoperative visual field deficit. ${ }^{19,33}$ Duration of awake surgeries can be decreased, ${ }^{5}$ and DTI can also serve as a backup tool either when patients are unsuitable for awake craniotomies or when an awake surgery must convert to "asleep" surgery for fatigue or seizure. ${ }^{25}$

DTI can occasionally prognosticate by quantifying a tumor's juxtaposition with or level of infiltration of relevant tracts. Jellison et al. described 4 categories of tumor effect on white matter tracts: 1) bulk mass displacement (FA normal, direction abnormal); 2) vasogenic edema (FA decreased, location/direction normal); 3) infiltration by the glioma (FA decreased, hues abnormal); and 4) total disruption (isotropic map, no tract identification). ${ }^{41}$ Affirming this concept, a small study of 12 right-handed patients undergoing craniotomy for tumors involving the left AF found that higher FA values were associated with improved long-term scores on the Western Aphasia Battery. ${ }^{47}$

More recently, the University of California, San Francisco group applied Q-ball tracking to assign quantitative measures of tract involvement in patients with newly diagnosed left hemispheric gliomas, both LGGs and HGGs. These investigators created a measurement called altered fiber tractography density (AFTD) and performed binary categorization as "affected" or "not affected" by summing points for the qualitative type of fiber effect. Postsurgery, predischarge tractography correlated to a positive predictive value and sensitivity of $86 \%(p=0.005)$ for the ability to predict long-term clinical language deficit. ${ }^{15}$ This valuable study correlating tract injury with postoperative outcomes expands the value of tractography beyond preoperative planning to include postoperative assessment, which in the future could be applied to connectomic assessments. Although it is a promising proof-of-concept study, the work needs to be validated in a much larger study, possibly a multicenter consortium.

DTI helps estimate the likelihood of a GTR (Tables 2 and 3). Castellano et al. retrospectively evaluated patients with glioma and dysembryoplastic neuroepithelial tumor for whom they used intraoperative stimulation and also constructed the CST, SLF, and IFOF by using standard DTI acquisitions. ${ }^{13}$ Their analysis revealed that DTI reconstruction of intact fascicles-as categorized by 2 neuroradiologists using Jellison and colleagues' scheme ${ }^{41}$ of assessing tract anisotropy, location, and orientation-accurately predicted the higher probability of total resection, whereas detection of infiltrated or displaced fascicles predicted a lower likelihood of total resection. ${ }^{13}$

Lesion-to-tract distance-the distance between tumor and 3 DTI tracts (SLF, cingulum, CST) - has been reviewed in 60 brain tumor cases, mostly glioma, in which a lesion-to-tract distance of $\leq 1 \mathrm{~cm}$ predicted an increase in postoperative deficit and mortality rates. ${ }^{55}$ Other studies support the accuracy of tractography-to within $1 \mathrm{~cm}$ of positive direct stimulation points. ${ }^{7,44,56}$

\section{Limitations of DTI}

Discordance between tractography and intraoperative direct stimulation, the reference gold standard, may result from accumulation of errors at each stage of DTI, beginning from MR distortions during the acquisition of the tensor information all the way to registration during surgery. False negatives can occur in tractography (a fiber can be missed) or false positives can occur, and it is difficult to control for them. ${ }^{52}$ Underlying anatomical differences in fibers, including simply their size, can translate into different FA values. Neurosurgical lesions incur edema and displace tracts. Functional reorganization can also occur. ${ }^{31}$ Seeding strategy introduces biases of density and volume in the reconstruction of tracts. For example, seeding from white matter of longer bundles results in estimations of greater tract density because more streamlines are initiated in longer than in shorter bundles. At the same time, longer bundles may experience a greater number of premature truncations. Some studies have compared the agreement with electrostimulation mapping and presurgical DTI for language tracts, finding that functional tracts in patients with glioma can be silent on preoperative imaging. ${ }^{50} \mathrm{~A}$ concern also arises that tractography can identify a tract that has in fact been made redundant by the effects of a nearby glioma, leading the surgeon to perform a subtotal resection, or even dissuading the surgeon entirely from tackling the tumor.

\section{Progress in Tractography \\ Edema Correction}

Edema surrounding tumors reduces the ability of early DTI methods to perform peritumoral tracking because the signal from free water competes with the signal from white matter. In these cases, the clinician can locally decrease FA threshold values-e.g., from 0.15 to 0.10 - to determine if additional tracts are included, but this step adds time and inconsistency. Fortunately, algorithms that facilitate edema invariance through multicompartment modeling of free water have emerged with improved peritumoral tracking $28,35,39,51$ (Fig. 1). Their incorporation into clinical software varies.

\section{Automated Tract Selection}

Recognizing neurosurgeons' challenge in selecting the appropriate tractography method in the absence of a verifiable "ground truth" standard, the medical image computing community identified a common set of cases involving the pyramidal tract on which to base the "DTI Challenge" in 2015. A panel of 5 judges qualitatively reviewed the final constructions of the international teams based on the presence of false-positive and false-negative tracts, as well as anatomical accuracy. ${ }^{63}$ Results from 8 teams demonstrated the heterogeneity in tract reconstruction that is showcased each time methodologies are compared with one another. ${ }^{52}$ One reason for variability is that tractography requires ROI placement, making it subjective, underlining the need for automated tract extraction.

Automated whole-brain tractography (AWBT) is now available to neurosurgeons who might not otherwise have time for planning or ready access to specialized neuroradiological tractography support ${ }^{54,58,59}$ (Fig. 3). Tunç et al. re- 
TABLE 2. Studies correlating extent of resection (GTR) with and without DTI

\begin{tabular}{|c|c|c|c|c|c|}
\hline Authors \& Year & No. of Pts & DTI Method & $\%$ w/ DTI & $\%$ w/o DTI & Location/Notes \\
\hline Chen et al., 2007 & $\begin{array}{l}7 \text { cavernomas, } 3 \\
\text { grade II gliomas }\end{array}$ & $\begin{array}{l}\text { Brainlab iPlan 2.5; } 6 \text { gradi- } \\
\quad \text { ent directions, } b=1000 \\
\text { sec/mm² }\end{array}$ & $60 \%$ & NA & Pontine lesions; DTI used in all cases w/ "great value" \\
\hline Wu et al., 2007 & 118 w/ DTI, 120 w/0 & $\begin{array}{l}\text { MRI protocols changed } \\
\text { w/in the study; } 6 \text { or } 25 \\
\text { gradient directions }\end{array}$ & $72 \%$ & $52 \%$ & Gliomas involving pyramidal tract, $p=0.002$ \\
\hline $\begin{array}{l}\text { D'Andrea et al., } \\
2012\end{array}$ & $\begin{array}{l}14 \text { glioma, } 4 \text { metas- } \\
\text { tases }\end{array}$ & $\begin{array}{l}\text { Brainlab iPlan 2.6; } 12 \\
\text { gradient directions, } b= \\
1000 \mathrm{sec} / \mathrm{mm}^{2}\end{array}$ & $100 \%$ & NA & $\begin{array}{l}\text { All tumors involved CST; multimodal adjuncts used } \\
\text { included intraop MRI after dural opening, neuro- } \\
\text { navigation, intraoperatively acquired tractography, } \\
\text { electrical mapping }\end{array}$ \\
\hline lus et al., 2012 & 190 total, 117 w/ DTI & Not specified & $90 \%$ & $77 \%$ & $\begin{array}{l}\text { LGGs involving eloquent areas; DTI cohort included } \\
\text { fMRI data }\end{array}$ \\
\hline
\end{tabular}

ported on the strong concordance between expert-defined tracts and connectivity-based clustering atlas-defined tracts in 10 patients. ${ }^{70}$ O'Donnell et al. applied automation retrospectively to brain tracts generated through a highly sensitive 2-tensor tractography method for 18 consecutive patients with brain tumors, finding that all functional MRI (fMRI) activation sites for language and motor localized to within $3 \mathrm{~mm}$ of corresponding tracts generated by automation. ${ }^{59}$ This concept has been validated against expert ROI placement in a cohort of 30 patients with temporal lobe epilepsy. ${ }^{54}$ Robust open-source options such as SlicerDMRI provide features and platforms that may facilitate cross-institutional work, as attested to by more than 75,000 downloads per year. ${ }^{58}$

\section{TABLE 3. Glossary of terms}

\begin{tabular}{|c|c|c|}
\hline $\begin{array}{l}\text { Acronym or } \\
\text { Term }\end{array}$ & Expanded Term & Explanation \\
\hline CSD & Constrained spherical deconvolution & $\begin{array}{l}\text { Uses HARDI to generate ODF, allowing visualization of multiple crossing fibers (e.g., lateral } \\
\text { homunculus) }\end{array}$ \\
\hline DSI & Diffusion spectrum imaging & $\begin{array}{l}b \text { typically } \geq 8000 \mathrm{sec} / \mathrm{mm}^{2} ; \text { uses } \geq 200 \text { gradient directions; provides subvoxel resolution; principle- } \\
\text { based; long scan time can render clinically prohibitive }{ }^{72}\end{array}$ \\
\hline DTI & Diffusion tensor imaging & $\begin{array}{l}\text { b typically } \geq 1000 \mathrm{sec} / \mathrm{mm}^{2} ; \text { requires } \geq 6 \text { gradient directions; relatively fast, automated postpro- } \\
\text { cessing is available; hardware well adapted to use; provides limited information about complex } \\
\text { architecture }\end{array}$ \\
\hline GQI & Generalized Q-sampling imaging & Model-free approach using Fourier transform to produce SDF from QBI or DSI17,72 \\
\hline HARDI & $\begin{array}{l}\text { High angular resolution diffusion } \\
\text { imaging }\end{array}$ & $\begin{array}{l}\text { Increased diffusion-weighted direction sampling (e.g., } 55 \text { gradient directions), stronger diffusion } \\
\left.\text { weighting (e.g., } b=2000 \mathrm{sec} / \mathrm{mm}^{2}\right) \text {; using GQI, provides subvoxel resolution for crossing fibers; } \\
\text { improves resolution of lateral tracks (e.g., centrum semiovale); cannot quantitatively define tis- } \\
\text { sue properties; longer acquisition times can be reduced by parallel imaging \& in a higher-tesla } \\
\text { MRI; eddy-current distortions more prevalent }{ }^{69}\end{array}$ \\
\hline HDFT & High-definition fiber tractography & $\begin{array}{l}\text { Non-gaussian, non-tensor approach permitting visualization of crossing fibers using GQl; re- } \\
\text { stricted \& nonrestricted diffusion indices, \& QA can be obtained }{ }^{61}\end{array}$ \\
\hline ODF & Orientation distribution function & $\begin{array}{l}\text { Generated from HARDI \& DSI using spherical deconvolution technique to resolve crossing fibers } \\
\text { w/in a voxe }{ }^{6}\end{array}$ \\
\hline QA & Quantitative anisotropy & $\begin{array}{l}\text { Quantification of the amount of anisotropic spins that diffuse along the fiber orientation, used as a } \\
\text { truncation threshold in streamlining algorithms }{ }^{61}\end{array}$ \\
\hline QBI & Q-ball imaging & $\begin{array}{l}b \text { typically }>4000 \mathrm{sec} / \mathrm{mm}^{2}, \& \text { at least } 60 \text { gradient directions are acquired; clinically feasible \& } \\
\text { provides good depiction of fiber crossing }\end{array}$ \\
\hline Seed point & Seed point & A specified point from which an algorithm is asked to track fibers bidirectionally ${ }^{42}$ \\
\hline $\mathrm{ROI}$ & Region of interest & $\begin{array}{l}\text { A user-defined planar or } 3 \mathrm{D} \text { region used to include or exclude traversing fibers when extracting } \\
\text { fiber bundles of interest }{ }^{42}\end{array}$ \\
\hline SDF & Spin distribution function & $\begin{array}{l}\text { Compound metric produced from GQI allowing multiple peaks of directionality w/in a single voxel; } \\
\text { index is } Q A^{61}\end{array}$ \\
\hline UKF & Unscented Kalman filter & $\begin{array}{l}\text { Applies recursive estimation \& filter framework to at least } 2 \text { tensors per voxel for better tracking of } \\
\text { crossing fibers }\end{array}$ \\
\hline
\end{tabular}




\section{Non-DTI Methods}

Although DTI became the standard clinical paradigm, high angular resolution diffusion imaging (HARDI) has been described since at least 2002, when it was developed to overcome the "crossing fiber problem"-intravoxel fiber orientational heterogeneity. ${ }^{69}$ By sampling increased numbers of diffusion-weighted directions rather than the minimum of 6, by increasing the diffusion weighting above $b$ $=1800 \mathrm{sec} / \mathrm{mm}^{2}$ to increase contrast for fiber resolution, and by increasing the angular resolution to increase perspective from multiple gradient orientations, 2 crossing fibers within a single voxel could be separately resolved. The HARDI method thus helped solve situations of oblateness, a measure scaling the difference between the second and third eigenvalues, which does not readily fit a gaussian model in many circumstances, but yet which contains important clues to the underlying white matter directionality. ${ }^{69}$

Diffusion spectrum imaging (DSI), another technique, requires tremendous data acquisition yet yields the greatest detail about brain architecture. In summary, the Fourier transform calculates from the succession of gradient pulses, each of which assesses the brain from a slightly different strength and direction, the diffusion probability density function for every voxel. Research progress has significantly diminished the time required for obtaining this in a patient. ${ }^{72}$

One method of using HARDI data to track fibers is to reconstruct it with a mathematical approach called Q-ball imaging (QBI). QBI attempts to combine the best of DTI (its speed and simplicity) with the best of HARDI and DSI (their resolution). QBI is a hypothesis-based simplification used to shorten acquisition time. It takes the data, such as from DSI or HARDI, with high $b$ values and high angular resolution, uses a mathematical entity called the Funk-Radon transform, takes certain liberties in assuming uniform diffusion along the length of impermeable pipes, and then determines overall directionality of the component pipes by sampling along the equators of given pipelines. From QBI an orientation distribution function (ODF) is derived, from which multiple subvoxel fiber populations are identified. ${ }^{6}$ In studies that used HARDI and QBI, surgeons have correlated strong intraoperative anatomical agreement. ${ }^{16}$ QBI simulates additional HARDI acquisitions according to a harmonic, and in conjunction with a QBI method called "residual bootstrap" characterizes the uncertainty in diffusion MR and the ODF shape-even from a single HARDI data set-allowing the detection of fibers that have traditionally been more difficult to visualize, like parts of the corpus callosum and lateral CSTs. As in all HARDI techniques, the robustness derives from bypassing the assumption of gaussian diffusion (on which DTI is based), but requires vast data acquisition.

High-definition fiber tractography (HDFT) is another advanced method of tractography allowing subvoxel resolution. Using a non-gaussian approach, HDFT does not identify tracts based on the directionality of voxel-sized tensors, but rather uses generalized Q-sampling imaging (GQI) to produce spin distribution functions (SDFs), which then produces an index of quantitative anisotropy (QA). Instead of correlating FA thresholds with fiber termination, HDFT uses QA as the index on which probabi- listic or deterministic tracking algorithms map out tracts. ${ }^{61}$ In HDFT, QA is a quantitative parameter that can be compared to the contralateral side for information that may potentially prognosticate. ${ }^{17,61}$

Constrained spherical deconvolution (CSD) is yet another methodology with the ability to discriminate 2 fiber populations within a single voxel. A head-to-head comparison against both deterministic and probabilistic DTI methods showed the superiority of CSD, with a probabilistic algorithm, to visualize the fan-shaped configuration of cortical spinal fibers close to the cortex. ${ }^{34}$ Furthermore, this advanced method can be supported with the opensource software MRTrix. ${ }^{67}$

Imaging performed at advanced centers using innovative, often proprietary, techniques like GQI provides more preoperative information, and this information enables a detailed, superior assessment of tract disruption by gliomas. One group related QA to the degree of peritumoral fiber tract involvement, finding it to be a biomarker of fiber tract integrity. ${ }^{17}$ Using GQI, they performed tractography on 16 patients with LGG who had never undergone surgery, finding evidence in favor of reserving awake craniotomies for patients with tract infiltration, sparing those with either pure displacement or pure disruption from the awake approach. Although it is tantalizing that this advanced fiber tracking and analysis provided guidance during resection, the QA approach toward tract assessment seems to have requirements that limit widespread adoption, including length of scan time (reported as 24 minutes) and per-subject basis adjustment of termination thresholds. ${ }^{17}$

The 2-tensor unscented Kalman filter (UKF) tractography technique recognizes that tracking from one voxel to the next is a causal process, so therefore by using recursive estimation, UKF resolves crossing fibers in peritumoral regions. The model breaks from single tensor tractography by operating within a filter framework to take the previous voxel information into account, and it applies 2 tensors at each voxel. ${ }^{53}$ Retrospectively, Chen et al. tracked the AF through edema in 10 patients with brain tumor, finding that UKF illuminated more than 4 times the volume of arcuate fibers as detected by conventional single tensor tractography. ${ }^{20}$

Ultimately, for the widespread adoption of next-generation tractography to occur, neurosurgeons must master awareness of the limitations of the current technology and request the best available technology. This enthusiasm could be directed toward a prospective, multiinstitutional effort to validate the most advanced imaging methods for sensitivity, specificity, and clinical relevance.

\section{Future Directions in Tractography for Glioma Surgery}

Studies have integrated fMRI with tractography, and another recently proposed method of integrating functionally validated data with tractography has been preoperative motor mapping performed using navigated transcranial magnetic brain stimulation (nTMS), which identified seed regions that were followed by tractography. ${ }^{48}$ This method improved resection and reduced deficits. Navigated transcranial magnetic brain stimulation has also been used to establish seeding points for tractography-based connectomics in glioma patients with aphasia. ${ }^{23}$ 

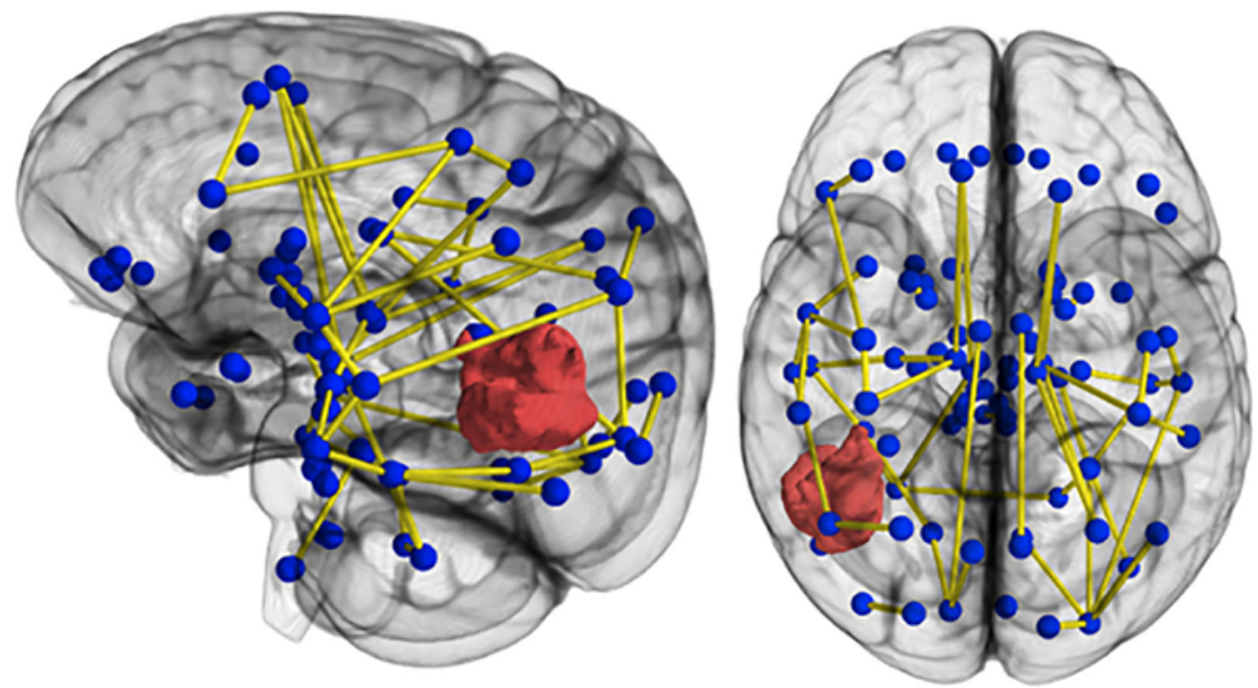

FIG. 5. Connectomics uses tractography to visualize human brain networks composed of white matter connections, modeled here as graph edges spanning between functional centers or hubs. Information contained within connectomes of patients with glioma may expand the definition of eloquence beyond discrete anatomical locations to include pathways participating in vital networks.

\section{Preoperative Assessment of the Connectome}

Twentieth-century research interpreted the brain with a "localizationist view of cerebral organization,"30 whereas now a "hodological" interpretation explains brain function in terms of parallel processing performed by distributed groups of connected and synchronized neurons rather than individual centers. ${ }^{26}$ Duffau proposed that surgical neurooncology should not be conceived as a focal surgery but as a brain network surgery. ${ }^{30}$

Brain connectomes can be modeled using their similarity to numerous interwoven "small world networks," being organized as specialized regions highly connected to neighbors but sparsely connected to distant regions. ${ }^{38}$ It has been well established that gliomas alter functional brain networks, and the more disruption, the worse the cognitive status. ${ }^{38,71,74}$ In short, glioma is not a focal disease ${ }^{27}$ but rather a network-related disease ${ }^{1}$ in which the connectome appears to contain molecular signatures for clinically relevant functionality 29,46 (Fig. 5). Preoperative assessment of the connectomic profile - of which tractography is a vital component-may increasingly predict the clinical phenotype with respect to survival and performance status. ${ }^{27}$

\section{Conclusions}

Tractography contributes valuable information about the relationship of gliomas to peritumoral tracts. Despite the limitations of DTI, its penetrance into neurosurgical practice surpasses that of higher-resolution diffusion and tractography algorithms. Furthermore, it has been difficult to robustly validate non-tensor modalities, hampering the justification of post-DTI alternatives. Ultimately, diffusion tractography aids neurosurgeons by revealing qualitative lines of maximum diffusion coherence, not quantitative axonal architecture, and as such its results will need to be interpreted with caution. ${ }^{42}$ Based on the emerging science of connectomics, which is linked to DTI, but encompass- ing brain networks, it is possible that we can stratify risk, link white matter pathology to clinical deficits, and monitor neuroplasticity and recovery of function, as well as use connectomics as a clinically relevant and potentially sensitive, noninvasive biomarker.

Consensus over which methodology should supplant DTI, or which improvement in its accuracy and utility should be adopted, will come with collaborative validation studies as well as further dialog between surgeons and industry partners. Tractography in its current state, although augmented by advances in edema invariance and tract automation, must be complemented by anatomical and electrophysiological methods. As neurosurgeons we have become interested in understanding and leveraging the "functional resilience of brain networks to pathological attack," 2 and we anticipate the increasing importance of a personalized understanding of each patient's network as a component of surgical planning. The incorporation of DTI into neurosurgical oncology practice has catalyzed important research into better tractography techniques that appear to minimize the functional consequences of surgery via preservation of functional networks.

\section{Acknowledgments}

The current work is supported in part by an NIH grant (RO1 NS096606; Principal Investigators R. Verma and S. Brem).

\section{References}

1. Aerts H, Fias W, Caeyenberghs K, Marinazzo D: Brain networks under attack: robustness properties and the impact of lesions. Brain 139:3063-3083, 2016

2. Bassett DS, Bullmore E: Small-world brain networks. Neuroscientist 12:512-523, 2006

3. Behrens TE, Berg HJ, Jbabdi S, Rushworth MF, Woolrich MW: Probabilistic diffusion tractography with multiple fibre orientations: what can we gain? Neuroimage 34:144-155, 2007 
4. Bello L, Castellano A, Fava E, Casaceli G, Riva M, Scotti $\mathrm{G}$, et al: Intraoperative use of diffusion tensor imaging fiber tractography and subcortical mapping for resection of gliomas: technical considerations. Neurosurg Focus 28(2):E6, 2010

5. Bello L, Gambini A, Castellano A, Carrabba G, Acerbi F, Fava E, et al: Motor and language DTI fiber tracking combined with intraoperative subcortical mapping for surgical removal of gliomas. Neuroimage 39:369-382, 2008

6. Berman J: Diffusion MR tractography as a tool for surgical planning. Magn Reson Imaging Clin N Am 17:205-214, 2009

7. Berman JI, Berger MS, Chung SW, Nagarajan SS, Henry RG: Accuracy of diffusion tensor magnetic resonance imaging tractography assessed using intraoperative subcortical stimulation mapping and magnetic source imaging. J Neurosurg 107:488-494, 2007

8. Bonney PA, Conner AK, Boettcher LB, Cheema AA, Glenn CA, Smitherman AD, et al: A simplified method of accurate postprocessing of diffusion tensor imaging for use in brain tumor resection. Oper Neurosurg (Hagerstown) 13:47-59, 2017

9. Briggs RG, Conner AK, Baker CM, Burks JD, Glenn CA, Sali G, et al: A Connectomic Atlas of the Human Cerebrum-Chapter 18: The Connectional Anatomy of $\mathrm{Hu}-$ man Brain Networks. Oper Neurosurg (Hagerstown) 15 (suppl_1):S470-S480, 2018

10. Briggs RG, Conner AK, Rahimi M, Sali G, Baker CM, Burks JD, et al: A Connectomic Atlas of the Human CerebrumChapter 14: Tractographic Description of the Frontal Aslant Tract. Oper Neurosurg (Hagerstown) 15 (suppl_1):S444S449, 2018

11. Briggs RG, Conner AK, Sali G, Rahimi M, Baker CM, Burks JD, et al: A Connectomic Atlas of the Human CerebrumChapter 17: Tractographic Description of the Cingulum. Oper Neurosurg (Hagerstown) 15 (suppl_1):S462-S469, 2018

12. Burks JD, Bonney PA, Conner AK, Glenn CA, Briggs RG, Battiste JD, et al: A method for safely resecting anterior butterfly gliomas: the surgical anatomy of the default mode network and the relevance of its preservation. J Neurosurg 126:1795-1811, 2017

13. Castellano A, Bello L, Michelozzi C, Gallucci M, Fava E, Iadanza A, et al: Role of diffusion tensor magnetic resonance tractography in predicting the extent of resection in glioma surgery. Neuro Oncol 14:192-202, 2012

14. Catani M, Howard RJ, Pajevic S, Jones DK: Virtual in vivo interactive dissection of white matter fasciculi in the human brain. Neuroimage 17:77-94, 2002

15. Caverzasi E, Hervey-Jumper SL, Jordan KM, Lobach IV, Li $\mathrm{J}$, Panara V, et al: Identifying preoperative language tracts and predicting postoperative functional recovery using HARDI q-ball fiber tractography in patients with gliomas. J Neurosurg 125:33-45, 2016

16. Caverzasi E, Papinutto N, Amirbekian B, Berger MS, Henry RG: Q-ball of inferior fronto-occipital fasciculus and beyond. PLoS One 9:e100274, 2014

17. Celtikci P, Fernandes-Cabral DT, Yeh FC, Panesar SS, Fernandez-Miranda JC: Generalized q-sampling imaging fiber tractography reveals displacement and infiltration of fiber tracts in low-grade gliomas. Neuroradiology 60:267-280, 2018

18. Chen X, Weigel D, Ganslandt O, Buchfelder M, Nimsky C: Diffusion tensor imaging and white matter tractography in patients with brainstem lesions. Acta Neurochir (Wien) 149:1117-1131, 2007

19. Chen X, Weigel D, Ganslandt O, Buchfelder M, Nimsky C: Prediction of visual field deficits by diffusion tensor imaging in temporal lobe epilepsy surgery. Neuroimage 45:286-297, 2009
20. Chen Z, Tie Y, Olubiyi O, Rigolo L, Mehrtash A, Norton I, et al: Reconstruction of the arcuate fasciculus for surgical planning in the setting of peritumoral edema using two-tensor unscented Kalman filter tractography. Neuroimage Clin 7:815-822, 2015

21. Conner AK, Briggs RG, Rahimi M, Sali G, Baker CM, Burks JD, et al: A Connectomic Atlas of the Human CerebrumChapter 10: Tractographic Description of the Superior Longitudinal Fasciculus. Oper Neurosurg (Hagerstown) 15 (suppl_1):S407-S422, 2018

22. Conner AK, Briggs RG, Sali G, Rahimi M, Baker CM, Burks JD, et al: A Connectomic Atlas of the Human CerebrumChapter 13: Tractographic Description of the Inferior FrontoOccipital Fasciculus. Oper Neurosurg (Hagerstown) 15 (suppl_1):S436-S443, 2018

23. Crimi A, Wiestler B, Kirschke JS, Krieg SM: Pre-operarative multivariate connectome analysis for glioma patients. bioRxiv [epub ahead of print], 2018

24. D'Andrea G, Angelini A, Romano A, Di Lauro A, Sessa G, Bozzao A, et al: Intraoperative DTI and brain mapping for surgery of neoplasm of the motor cortex and the corticospinal tract: our protocol and series in BrainSUITE. Neurosurg Rev 35:401-412, 2012

25. D'Andrea G, Familiari P, Di Lauro A, Angelini A, Sessa G: Safe resection of gliomas of the dominant angular gyrus availing of preoperative FMRI and intraoperative DTI: preliminary series and surgical technique. World Neurosurg 87:627-639, 2016

26. De Benedictis A, Duffau H: Brain hodotopy: from esoteric concept to practical surgical applications. Neurosurgery 68:1709-1723, 2011

27. Derks J, Dirkson AR, de Witt Hamer PC, van Geest Q, Hulst $\mathrm{HE}$, Barkhof F, et al: Connectomic profile and clinical phenotype in newly diagnosed glioma patients. Neuroimage Clin 14:87-96, 2017

28. Deslauriers-Gauthier S, Parker D, Rheault F, Deriche R, Brem S, Descoteaux M, et al: Edema-informed anatomically constrained particle filter tractography, in Frangi AF, Schnabel JA, Davatzikos C, et al (eds): Medical Image Computing and Computer-Assisted Intervention-MICCAI 2018. Berlin: Springer, 2018, pp 375-382

29. Douw L, Miller JJ, Steenwijk MD, Stufflebeam SM, Gerstner ER: Altered structural hub connectivity and its clinical relevance in glioma. bioRxiv [epub ahead of print], 2019

30. Duffau H: Surgical neurooncology is a brain networks surgery: a "connectomic" perspective. World Neurosurg 82:e405-e407, 2014

31. Duffau H: The huge plastic potential of adult brain and the role of connectomics: new insights provided by serial mappings in glioma surgery. Cortex 58:325-337, 2014

32. Duffau H, Mandonnet E: The "onco-functional balance" in surgery for diffuse low-grade glioma: integrating the extent of resection with quality of life. Acta Neurochir (Wien) 155:951-957, 2013

33. Essayed WI, Zhang F, Unadkat P, Cosgrove GR, Golby AJ, O'Donnell LJ: White matter tractography for neurosurgical planning: a topography-based review of the current state of the art. Neuroimage Clin 15:659-672, 2017

34. Farquharson S, Tournier JD, Calamante F, Fabinyi G, Schneider-Kolsky M, Jackson GD, et al: White matter fiber tractography: why we need to move beyond DTI. J Neurosurg 118:1367-1377, 2013

35. Gong S, Zhang F, Norton I, Essayed WI, Unadkat P, Rigolo L, et al: Free water modeling of peritumoral edema using multi-fiber tractography: application to tracking the arcuate fasciculus for neurosurgical planning. PLoS One 13:e0197056, 2018

36. González-Darder JM, González-López P, Talamantes F, Quilis V, Cortés V, García-March G, et al: Multimodal navi- 
gation in the functional microsurgical resection of intrinsic brain tumors located in eloquent motor areas: role of tractography. Neurosurg Focus 28(2):E5, 2010

37. Hagmann P, Kurant M, Gigandet X, Thiran P, Wedeen VJ, Meuli R, et al: Mapping human whole-brain structural networks with diffusion MRI. PLoS One 2:e597, 2007

38. Huang Q, Zhang R, Hu X, Ding S, Qian J, Lei T, et al: Disturbed small-world networks and neurocognitive function in frontal lobe low-grade glioma patients. PLoS One 9:e94095, 2014

39. Ismail AAO, Parker D, Hernandez-Fernandez M, Wolf R, Brem S, Alexander S, et al: Freewater EstimatoR using iNtErpolated iniTialization (FERNET): toward accurate estimation of free water in peritumoral region using single-shell diffusion MRI data. Inserm-02309753. 2019 (https://www. hal.inserm.fr/inserm-02309753) [Accessed December 3, 2019]

40. Ius T, Isola M, Budai R, Pauletto G, Tomasino B, Fadiga L, et al: Low-grade glioma surgery in eloquent areas: volumetric analysis of extent of resection and its impact on overall survival. A single-institution experience in 190 patients: clinical article. J Neurosurg 117:1039-1052, 2012

41. Jellison BJ, Field AS, Medow J, Lazar M, Salamat MS, Alexander AL: Diffusion tensor imaging of cerebral white matter: a pictorial review of physics, fiber tract anatomy, and tumor imaging patterns. AJNR Am J Neuroradiol 25:356-369, 2004

42. Jeurissen B, Descoteaux M, Mori S, Leemans A: Diffusion MRI fiber tractography of the brain. NMR Biomed 32:e3785, 2019

43. Jones DK, Pierpaoli C: Confidence mapping in diffusion tensor magnetic resonance imaging tractography using a bootstrap approach. Magn Reson Med 53:1143-1149, 2005

44. Kamada K, Todo T, Masutani Y, Aoki S, Ino K, Takano T, et al: Combined use of tractography-integrated functional neuronavigation and direct fiber stimulation. J Neurosurg 102:664-672, 2005

45. Kamada K, Todo T, Ota T, Ino K, Masutani Y, Aoki S, et al: The motor-evoked potential threshold evaluated by tractography and electrical stimulation. J Neurosurg 111:785-795, 2009

46. Kesler SR, Noll K, Cahill DP, Rao G, Wefel JS: The effect of IDH1 mutation on the structural connectome in malignant astrocytoma. J Neurooncol 131:565-574, 2017

47. Kinoshita M, Nakada M, Okita H, Hamada J, Hayashi Y: Predictive value of fractional anisotropy of the arcuate fasciculus for the functional recovery of language after brain tumor resection: a preliminary study. Clin Neurol Neurosurg 117:45-50, 2014

48. Krieg SM, Sollmann N, Obermueller T, Sabih J, Bulubas L, Negwer C, et al: Changing the clinical course of glioma patients by preoperative motor mapping with navigated transcranial magnetic brain stimulation. BMC Cancer 15:231, 2015

49. Lazar M, Alexander AL: Bootstrap white matter tractography (BOOT-TRAC). Neuroimage 24:524-532, 2005

50. Leclercq D, Duffau H, Delmaire C, Capelle L, Gatignol P, Ducros M, et al: Comparison of diffusion tensor imaging tractography of language tracts and intraoperative subcortical stimulations. J Neurosurg 112:503-511, 2010

51. Lecoeur J, Caruyer E, Elliott M, Brem S, Macyszyn L, Verma $\mathrm{R}$ : Addressing the challenge of edema in fiber tracking, presented at the MICCAI 2014 DTI Tractography Challenge, September 2014 (Abstract) (https://hal.inria.fr/hal-01072222) [Accessed December 3, 2019]

52. Maier-Hein KH, Neher PF, Houde JC, Côté MA, Garyfallidis E, Zhong J, et al: The challenge of mapping the human connectome based on diffusion tractography. Nat Commun 8:1349, 2017
53. Malcolm JG, Shenton ME, Rathi Y: Filtered multitensor tractography. IEEE Trans Med Imaging 29:1664-1675, 2010

54. Mancini M, Vos SB, Vakharia VN, O'Keeffe AG, Trimmel $\mathrm{K}$, Barkhof F, et al: Automated fiber tract reconstruction for surgery planning: extensive validation in language-related white matter tracts. Neuroimage Clin 23:101883, 2019

55. Meyer EJ, Gaggl W, Gilloon B, Swan B, Greenstein M, Voss $\mathrm{J}$, et al: The impact of intracranial tumor proximity to white matter tracts on morbidity and mortality: a retrospective diffusion tensor imaging study. Neurosurgery 80:193-200, 2017

56. Mikuni N, Okada T, Enatsu R, Miki Y, Hanakawa T, Urayama S, et al: Clinical impact of integrated functional neuronavigation and subcortical electrical stimulation to preserve motor function during resection of brain tumors. J Neurosurg 106:593-598, 2007

57. Mukherjee P, Berman JI, Chung SW, Hess CP, Henry RG: Diffusion tensor MR imaging and fiber tractography: theoretic underpinnings. AJNR Am J Neuroradiol 29:632-641, 2008

58. Norton I, Essayed W, Zhang F, Pujol S, Yarmarkovich A, Golby AJ, et al: SlicerDMRI: open source diffusion MRI software for brain cancer research. Cancer Res 77:e101e103, 2017

59. O’Donnell LJ, Suter Y, Rigolo L, Kahali P, Zhang F, Norton I, et al: Automated white matter fiber tract identification in patients with brain tumors. Neuroimage Clin 13:138-153, 2016

60. Ohue S, Kohno S, Inoue A, Yamashita D, Harada H, Kumon Y, et al: Accuracy of diffusion tensor magnetic resonance imaging-based tractography for surgery of gliomas near the pyramidal tract: a significant correlation between subcortical electrical stimulation and postoperative tractography. Neurosurgery 70:283-294, 2012

61. Panesar SS, Abhinav K, Yeh FC, Jacquesson T, Collins M, Fernandez-Miranda J: Tractography for surgical neuro-oncology planning: towards a gold standard. Neurotherapeutics 16:36-51, 2019

62. Piper RJ, Yoong MM, Kandasamy J, Chin RF: Application of diffusion tensor imaging and tractography of the optic radiation in anterior temporal lobe resection for epilepsy: a systematic review. Clin Neurol Neurosurg 124:59-65, 2014

63. Pujol S, Wells W, Pierpaoli C, Brun C, Gee J, Cheng G, et al: The DTI Challenge: toward standardized evaluation of diffusion tensor imaging tractography for neurosurgery. J Neuroimaging 25:875-882, 2015

64. Sali G, Briggs RG, Conner AK, Rahimi M, Baker CM, Burks JD, et al: A Connectomic Atlas of the Human Cerebrum-Chapter 11: Tractographic Description of the Inferior Longitudinal Fasciculus. Oper Neurosurg (Hagerstown) 15 (suppl_1):S423-S428, 2018

65. Sanai $\mathbf{N}$, Berger MS: Intraoperative stimulation techniques for functional pathway preservation and glioma resection. Neurosurg Focus 28(2):E1, 2010

66. Sanai N, Berger MS: Surgical oncology for gliomas: the state of the art. Nat Rev Clin Oncol 15:112-125, 2018

67. Tournier JD, Smith R, Raffelt D, Tabbara R, Dhollander T, Pietsch M, et al: MRtrix3: A fast, flexible and open software framework for medical image processing and visualisation. Neuroimage 202:116137, 2019

68. Tuch DS: Q-ball imaging. Magn Reson Med 52:1358-1372, 2004

69. Tuch DS, Reese TG, Wiegell MR, Makris N, Belliveau JW, Wedeen VJ: High angular resolution diffusion imaging reveals intravoxel white matter fiber heterogeneity. Magn Reson Med 48:577-582, 2002

70. Tunç B, Ingalhalikar M, Parker D, Lecoeur J, Singh N, Wolf RL, et al: Individualized map of white matter pathways: connectivity-based paradigm for neurosurgical planning. Neurosurgery 79:568-577, 2016 
71. van Dellen E, de Witt Hamer PC, Douw L, Klein M, Heimans JJ, Stam CJ, et al: Connectivity in MEG resting-state networks increases after resective surgery for low-grade glioma and correlates with improved cognitive performance. Neuroimage Clin 2:1-7, 2012

72. Wedeen VJ, Wang RP, Schmahmann JD, Benner T, Tseng WY, Dai G, et al: Diffusion spectrum magnetic resonance imaging (DSI) tractography of crossing fibers. Neuroimage 41:1267-1277, 2008

73. Wu JS, Zhou LF, Tang WJ, Mao Y, Hu J, Song YY, et al: Clinical evaluation and follow-up outcome of diffusion tensor imaging-based functional neuronavigation: a prospective, controlled study in patients with gliomas involving pyramidal tracts. Neurosurgery 61:935-949, 2007

74. Xu H, Ding S, Hu X, Yang K, Xiao C, Zou Y, et al: Reduced efficiency of functional brain network underlying intellectual decline in patients with low-grade glioma. Neurosci Lett 543:27-31, 2013

75. Zhu FP, Wu JS, Song YY, Yao CJ, Zhuang DX, Xu G, et al: Clinical application of motor pathway mapping using diffusion tensor imaging tractography and intraoperative direct subcortical stimulation in cerebral glioma surgery: a prospective cohort study. Neurosurgery 71:1170-1184, 2012

\section{Disclosures}

Dr. Verma received clinical or research support for the study described (includes equipment or material) from Synaptive Medical Systems.

\section{Author Contributions}

Conception and design: all authors. Acquisition of data: all authors. Analysis and interpretation of data: all authors. Drafting the article: all authors. Critically revising the article: all authors. Reviewed submitted version of manuscript: all authors. Approved the final version of the manuscript on behalf of all authors: Brem. Study supervision: Verma, Brem.

\section{Correspondence}

Steven Brem: Perelman School of Medicine, University of Pennsylvania, Philadelphia, PA. steven.brem@pennmedicine. upenn.edu. 